\title{
Bruhat order on fixed-point-free involutions in the symmetric group
}

\author{
Matthew Watson* \\ Department of Mathematics \\ North Carolina State University \\ Raleigh, NC 27695, U.S.A. \\ mbwatso2@ncsu.edu
}

Submitted: Nov 6, 2013; Accepted: Mar 30, 2014; Published: May 2, 2014

Mathematics Subject Classifications: 05C88, 05C89

\begin{abstract}
We provide a structural description of Bruhat order on the set $F_{2 n}$ of fixed-pointfree involutions in the symmetric group $S_{2 n}$ which yields a combinatorial proof of a combinatorial identity that is an expansion of its rank-generating function. The decomposition is accomplished via a natural poset congruence, which yields a new interpretation and proof of a combinatorial identity that counts the number of rook placements on the Ferrers boards lying under all Dyck paths of a given length $2 n$. Additionally, this result extends naturally to prove new combinatorial identities that sum over other Catalan objects: 312-avoiding permutations, plane forests, and binary trees.
\end{abstract}

\section{Introduction}

There is a family of combinatorial identities that express sums over certain Catalan objects in nice closed forms. Perhaps one of the most notable members of this family is Postnikov's hook-length formula,

$$
\sum_{T \in \mathcal{B}_{n}} \prod_{v \in T}\left(\frac{h(v)+1}{h(v)}\right)=\frac{2^{n}(n+1)^{n-1}}{n !},
$$

which can be found in [9]. This sum is taken over the set $\mathcal{B}_{n}$ of binary trees with $n$ nodes, and the value $h(v)$ is the the number of descendants of the node $v$. Another member of

*Partially supported by NSF Grant 0943855. 
this family,

$$
n ! \sum_{F \in \mathcal{F}_{n}} \prod_{v \in F} \frac{1}{h(v)}=(2 n-1) ! !,
$$

appears in [5], which is a sum over the set $\mathcal{F}_{n}$ of plane forests with $n$ nodes. It is wellknown that the Catalan numbers enumerate $\mathcal{B}_{n}$ and $\mathcal{F}_{n}$ [13, Exercise 6.19]. We prove the following identity in this family:

$$
\sum_{\delta \in \mathcal{D}_{n}} r_{n}(B(\delta))=(2 n-1) ! !
$$

This sum is taken over the set $\mathcal{D}_{n}$ of Dyck paths of length $2 n$. The statistic $r_{n}(B(\delta))$ is the $n$th rook number of the Ferrers board $B(\delta) \subset[n] \times[n]$ lying under the Dyck path $\delta \in \mathcal{D}_{n}$. As an example, consider the Dyck paths in $\mathcal{D}_{3}$ shown in Figure 1 . The assertion made by (3) is that $1+2+2+4+6=5$ !!. Each summand is the number of (non-attacking) 3 -rook placements on the respective Ferrers boards.
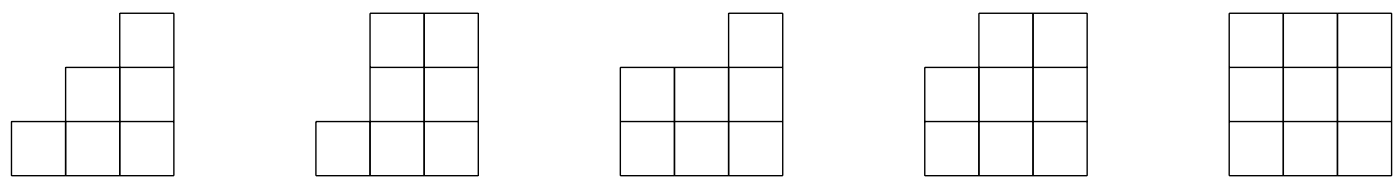

Figure 1: The Ferrers boards lying under the five Dyck paths in $\mathcal{D}_{3}$

Identity (3) is merely a consequence of our main focus, a combinatorial proof of Theorem 1 , which is a decomposition of the rank-generating function of the poset $F_{2 n}$ consisting of the fixed-point-free involutions in $S_{2 n}$ under Bruhat order.

Theorem 1. For all $n \in \mathbb{N}$,

$$
\sum_{\delta \in \mathcal{D}_{n}} \prod_{i \in[n]} q^{d_{i}(\delta)-2 i}\left[d_{i}(\delta)-2 i+1\right]_{q}=[1]_{q}[3]_{q} \cdots[2 n-1]_{q}
$$

Here, $d_{i}(\delta)$ is a statistic on the Dyck path $\delta \in \mathcal{D}_{n}$, which we describe in Section 3.1.1, and $\prod_{i \in[n]}\left[d_{i}(\delta)-2 i+1\right]_{q}$ is a $q$-analog of the rook number $r_{n}(B(\delta))$.

In order to dissect the poset $F_{2 n}$, we make use of a bijection $\varphi$ between fixed-point-free involutions and rook placements on Ferrers boards. This bijection is decribed specifically in Section 3.2, and it is well-known as a part of combinatorial folklore among those concerned with perfect matchings.

This realization leads to a decomposition of the poset into disjoint intervals, which in turn yields the expansion of the rank-generating function of $F_{2 n}$ given in Theorem 1. The decomposition of $F_{2 n}$ is accomplished via a poset congruence in the sense of [10]. (A poset congruence is the order-theoretic generalization of a lattice congruence.) Figure 2 illustrates the Hasse diagram of Bruhat order on $F_{6}$ under the bijection $\varphi$.

It should be mentioned that (4) appears in [1], in which the authors refer to it as a classical identity. This equation can be derived from [6, Exercise 5.2.9 (b)] using hypergeometric series. Our proof of this result is combinatorial, thus it is very different in nature. Moreover, it describes something fundamental about fixed-point-free involutions. 


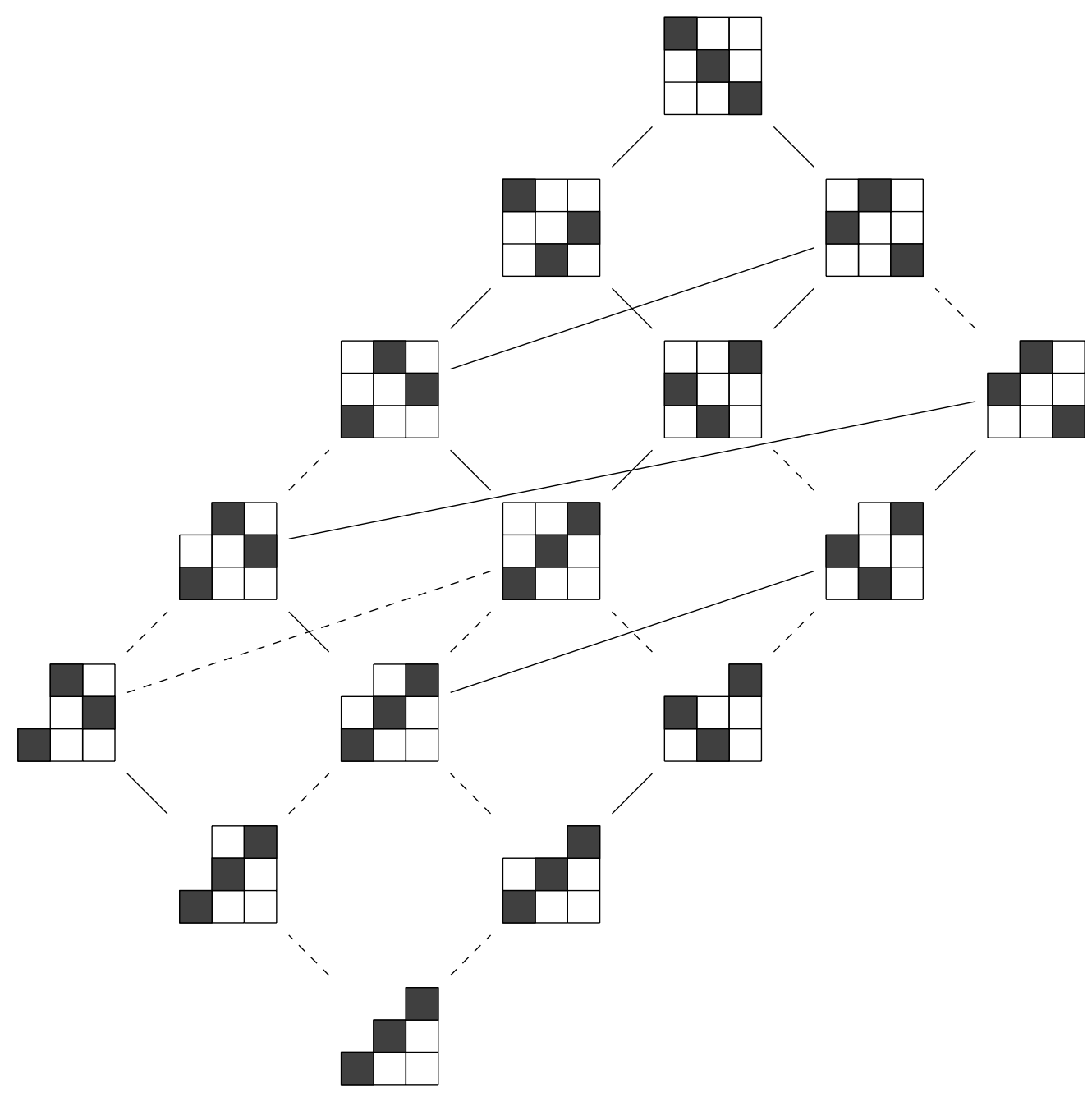

Figure 2: Bruhat order on $F_{6}$ represented as rook placements on Dyck boards

The connection between these Catalan objects highlights a sense in which binary trees relate to permutations as plane forests relate to fixed-point-free involutions. Specifically, there is a natural map $S_{n} \rightarrow \mathcal{B}_{n}$ (cf. [3], [11]) that is a lattice homomorphism of the weak order on $S_{n}$, and the natural partial order on the image is the quotient - the Tamari lattice. In particular, the fibers of the map constitute a lattice congruence on the weak order. In this paper, we exhibit a natural map $F_{2 n} \rightarrow \mathcal{F}_{n}$ whose fibers constitute a poset congruence on the Bruhat order on $F_{2 n}$. The quotient modulo this congruence is a natural partial order on Dyck paths.

We extend this enumerative result to the context of plane forests and binary trees. In particular, under two natural bijections, the rook number statistic of each Dyck path is equivalent to statistics on these objects. This yields additional identities in the family of Catalan sums. 


\section{Fixed-point-free involutions}

The objects of concern to us are elements of the symmetric group, thus we begin by recalling pertinent notions relating to the symmetric group and Bruhat order in Section 2.1. Section 2.2 contains a characterization of the set $F_{2 n}$ of fixed-point-free involutions, and we describe in Section 2.3 the subposet of these permutations under the order induced by Bruhat order on $S_{2 n}$.

\subsection{The symmetric group}

For $n \geqslant 1$, let $S_{n}$ denote the symmetric group, which consists of all permutations $\sigma:[n] \rightarrow[n]$, where $[n]$ denotes the set $\{1,2, \ldots, n\}$. Each permutation $\sigma \in S_{n}$ can be written in one-line notation as $\sigma_{1} \sigma_{2} \cdots \sigma_{n}$, where $\sigma_{i}:=\sigma(i)$ for all $i \in[n]$. Alternatively, $\sigma \in S_{n}$ can be decomposed into its cycle notation in which it is written as a product of unique disjoint cycles of the form $\left(\sigma_{i} \sigma_{i}^{2} \cdots \sigma_{i}^{k}\right)$, in which $\sigma_{i}^{k+1}=\sigma_{i}$. We omit 1-cycles from cycle notation. The canonical cycle notation of a permutation is its cycle decomposition with its cycles in ascending order by their minimal elements and the elements within cycles arranged so that the least value appears first. We use the notation $\left(\sigma_{i} \sigma_{i}^{2} \cdots \sigma_{i}^{k}\right) \in \sigma$ to denote that the cycle $\left(\sigma_{i} \sigma_{i}^{2} \cdots \sigma_{i}^{k}\right)$ appears in the canonical cycle notation of $\sigma$. We denote by $i d_{n}$ the identity permutation $12 \cdots n$ in $S_{n}$.

The permutation diagram of $\sigma \in S_{n}$ is the $n \times n$ array of squares, which we call cells, with the set $\left\{\left(i, \sigma_{i}\right): i \in[n]\right\} \subset[n] \times[n]$ of cells shaded. The cell $(i, j)$ is in the $i$ th column from the left and the $j$ th row from the bottom.

Example 2. The permutation diagram of $\sigma=416523 \in S_{6}$ is pictured in Figure 3(a).

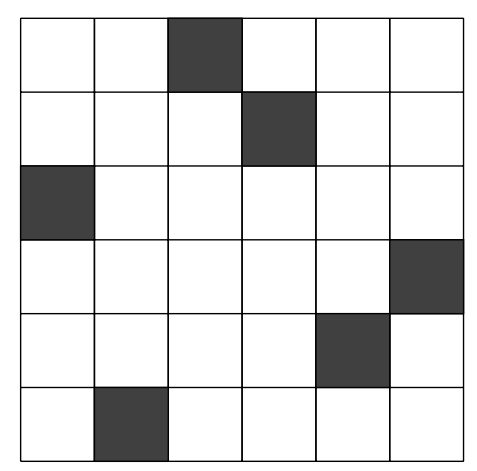

(a) $416523 \in S_{6}$

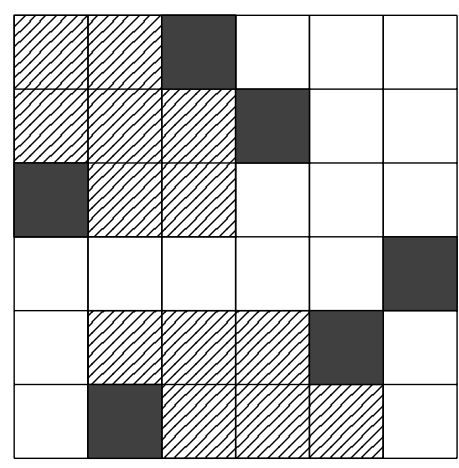

(b) Two free rises of $416523 \in S_{6}$

Figure 3

An inversion of $\sigma \in S_{n}$ is a pair $\left(\sigma_{i}, \sigma_{j}\right) \in[n]^{2}$ such that $i<j$ and $\sigma_{i}>\sigma_{j}$. The inversion number $\operatorname{inv}(\sigma)$ of $\sigma$ is the number of its inversions. A rise of $\sigma \in S_{n}$ is a pair $(i, j) \in[n]^{2}$ such that $i<j$ and $\sigma_{i}<\sigma_{j}$. A rise is called free if there exists no $k \in[n]$ such that $i<k<j$ and $\sigma_{i}<\sigma_{k}<\sigma_{j}$. Each $i \in[n]$ satisfies exactly one of the following 
relations: $\sigma_{i}<i ; \sigma_{i}>i$; or $\sigma_{i}=i$. In these cases, $i$ is called a deficiency, an exceedance, or a fixed-point of $\sigma$, respectively.

Bruhat order on $S_{n}$ is the partial order relation $\leqslant_{B}$ which is the transitive closure of the relation $\rightarrow$ defined by $\sigma \rightarrow \tau$ if and only if $\sigma(i j)=\tau$ for some transposition $(i j)$ and $\operatorname{inv}(\sigma)<\operatorname{inv}(\tau)$. Let $\triangleleft_{B}$ denote the covering relation in Bruhat order. The rank function of $S_{n}$ under Bruhat order is given by $\operatorname{inv}(\sigma)$ for all $\sigma \in S_{n}$. The following descriptions of Bruhat order will be useful.

Theorem 3. [2, Theorem 2.1.1] For all $\sigma \in S_{n}$ and $(h, k) \in[n]^{2}$, define

$$
\sigma[h, k]:=\left|\left\{i \in[h]: \sigma_{i} \geqslant k\right\}\right| .
$$

If $\sigma, \tau \in S_{n}$, then $\sigma \leqslant_{B} \tau$ if and only if $\sigma[h, k] \leqslant \tau[h, k]$ for all $(h, k) \in[n]^{2}$.

Theorem 4. [7, Theorem 5.1] Suppose $\sigma, \tau \in S_{n}$. Then $\sigma \triangleleft_{B} \tau$ if and only if $\tau=\sigma(i j)$ for some free rise $(i, j)$ of $\sigma$.

Given permutations $\sigma \in S_{n}$ and $\tau \in S_{m}$, we say that $\sigma$ contains $\tau$ if there exist $1 \leqslant i_{1}<i_{2}<\cdots<i_{m} \leqslant n$ such that $\sigma_{i_{j}}<\sigma_{i_{k}}$ if and only if $\tau_{j}<\tau_{k}$ for every pair $j, k \in[m]$. If $\sigma$ does not contain $\tau$, then we say $\sigma$ is $\tau$-avoiding. Let $S_{n}(\tau)$ denote the set of $\tau$-avoiding permutations in $S_{n}$. It is well-known [8] that the $n$th Catalan number $C_{n}$ is the number of permutations in $S_{n}(\tau)$ for any $\tau \in S_{3}$.

Example 5. The fourteen 312-avoiding permutations in $S_{4}$ are 1234, 1243, 1324, 1342, 1432, 2134, 2143, 2314, 2341, 2431, 3214, 3241, 3421, and 4321.

\subsection{The set $F_{2 n}$}

Let $F_{2 n}$ denote the set of fixed-point-free involutions in $S_{2 n}$. Note that any $x \in F_{2 n}$ has $n$ transpositions in its canonical cycle notation $\left(e_{1} d_{1}\right)\left(e_{2} d_{2}\right) \cdots\left(e_{n} d_{n}\right)$. Additionally, we have $e_{i}<d_{i}$ for all $i \in n$ and $e_{i}<e_{i+1}$ for all $i \in[n-1]$. We use the characters $e$ and $d$ to indicate which values are exceedances and which are deficiencies. The cardinality of $F_{2 n}$ is $(2 n-1) !$ !.

Being composed as a set of ordered pairs lends itself to a more pictorial representation: the arc diagram of $x \in F_{2 n}$ is a directed complete matching on the node set $[2 n]$ with edge set $\{(i, j):(i j) \in x\}$, where $(i, j)$ denotes the directed edge from the initial point $i$ to the terminal point $j$.

Example 6. Figure 4 shows the arc diagram of $73286514 \in F_{8}$.

We adopt from [4] the following definitions relating to the arc diagram of a fixedpoint-free involution. Suppose $x \in F_{2 n}$ and $\left(e_{i} d_{i}\right),\left(e_{j} d_{j}\right) \in x$ for $i<j$. The two arcs $\left(e_{i} d_{i}\right),\left(e_{j} d_{j}\right) \in x$ are crossing if $e_{i}<e_{j}<d_{i}<d_{j}$. When no pair of arcs of an arc diagram are crossing, we say the arc diagram is non-crossing. It is well-known that the $n$th Catalan number $C_{n}$ is the number of non-crossing arc diagrams on $2 n$ vertices [13, Exercise. 6.19(o)]. Define the crossing number $\operatorname{cross}(x)$ of $x \in F_{2 n}$ to be the number of pairs of arcs in its arc diagram that are crossing. Define $\operatorname{span}(x):=\sum_{(e d) \in x} \operatorname{span}(e, d)$, where $\operatorname{span}(e, d):=d-e-1$, and define the weight of $x \in F_{2 n}$ to be $\operatorname{wt}(x):=\operatorname{span}(x)-\operatorname{cross}(x)$. 


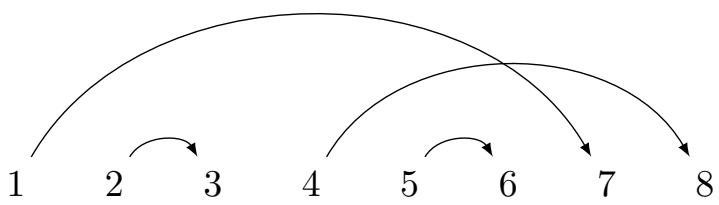

Figure 4: $(17)(23)(48)(56)=73286514 \in F_{8}$

Example 7. Consider the arc diagram of $x:=73286514 \in F_{8}$ shown in Figure 4 . The span of $x$ is 8 , and arcs (17) and (48) form the only crossing pair, thus wt $(x)=8$.

Proposition 8. For any $i<j$, the two arcs $\left(e_{i}, d_{i}\right)$ and $\left(e_{j}, d_{j}\right)$ cross in the arc diagram of $x \in F_{2 n}$ if and only if $e_{j}<i+j$ if and only if $i+j \leqslant d_{i}$.

Proof. By definition, the two arcs cross if and only if $e_{i}<e_{j}<d_{i}<d_{j}$. It is clear then that the crossing is due solely to the middlemost inequality, meaning that the $j$ th $e$ is preceded by strictly less than $i$ terminal points. Equivalently, the $i$ th $d$ is preceded by at least $j$ initial points. In other words, $e_{j}<i+j$ if and only if $i+j \leqslant d_{i}$.

Proposition 9. If $x \in F_{2 n}$, then $\operatorname{inv}(x)=2 \mathrm{wt}(x)+n$.

Proof. We proceed by induction, so first note that the proposition holds in the $n=1$ case. Now, consider $x \in F_{2 n}$ for some $n>1$, and suppose the proposition holds for $n-1$. By removing the arc $(i, 2 n)$ from the arc diagram of $x$ and subtracting 1 from the entries between $i$ and $2 n$, we obtain the arc diagram for $y \in F_{2 n-2}$. By the inductive hypothesis, $\operatorname{inv}(y)=2 \mathrm{wt}(y)+(n-1)$. Then, letting $c_{x}(a, b)$ denote the number of arcs crossing the arc $(a, b)$ in $x$, we have

$$
\operatorname{span}(x)=\operatorname{span}(y)+c_{x}(i, 2 n)+(2 n-i-1)
$$

and

$$
\operatorname{cross}(x)=\operatorname{cross}(y)+c_{x}(i, 2 n)
$$

Now,

$$
\begin{aligned}
\operatorname{wt}(x) & =\operatorname{span}(x)-\operatorname{cross}(x) \\
& =\operatorname{span}(y)+c_{x}(i, 2 n)+(2 n-i-1)-\operatorname{cross}(y)-c_{x}(i, 2 n) \\
& =\operatorname{span}(y)-\operatorname{cross}(y)+(2 n-i-1) \\
& =\operatorname{wt}(y)+(2 n-i-1)
\end{aligned}
$$

Thus

$$
\begin{aligned}
\operatorname{inv}(x) & =\operatorname{inv}(y)+(2 n-i-1)+(2 n-i) \\
& =2 \operatorname{wt}(y)+(n-1)+(2 n-i-1)+(2 n-i) \\
& =2 \operatorname{wt}(y)+n-1+2 n-i-1+2 n-i \\
& =2(\operatorname{wt}(y)+2 n-i-1)+n \\
& =2 \operatorname{wt}(x)+n,
\end{aligned}
$$

and the proposition holds for $n$. Thus the proposition holds for all natural numbers. 


\subsection{Bruhat order on $\boldsymbol{F}_{2 n}$}

Let the partial order relation $\leqslant$ be the restriction of Bruhat order on the elements of $F_{2 n}$, and let $\triangleleft$ denote the corresponding cover relation. Both the set $F_{2 n}$ and the poset $\left(F_{2 n}, \leqslant\right)$ will be denoted by $F_{2 n}$, as no confusion should arise. Because Bruhat order is graded by inversion number, Theorem 4 implies the following lemma.

Lemma 10. Suppose $x \in F_{2 n}$. If $(i, j)$ is a free rise of $x$ and $\left(x_{i}, x_{j}\right)$ is a free rise of $x(i j)$, then $\operatorname{inv}((i j) x(i j))=\operatorname{inv}(x)+2$.

Proposition 11. The cover relation $x \triangleleft y$ exists in $F_{2 n}$ if and only if $y=(i j) x(i j)$ for some free rise $(i, j)$ of $x$ such that $\operatorname{inv}(y)=\operatorname{inv}(x)+2$.

Proof. Let $x, y \in F_{2 n}$. Suppose $\operatorname{inv}(y)=\operatorname{inv}(x)+2$ and there exists some free rise $(i, j)$ of $x$ such that $y=(i j) x(i j)$. The existence of the cover relations $x \triangleleft_{B} x(i j)$ and $x(i j) \triangleleft_{B} y$ follow from Theorem 4 and the fact that $y=x(i j)\left(x_{i} x_{j}\right)$. Bruhat order is ranked by inversion number, and $\operatorname{inv}(y)=\operatorname{inv}(x)+2$, thus $x \triangleleft y$.

Conversely, suppose $x \triangleleft y$. Define

$$
i:=\min \left\{k \in[2 n]: x_{k} \neq y_{k}\right\}
$$

and

$$
j:=\min \left\{k \in[i+1,2 n]: x_{k} \leqslant y_{i} \text { and }(i, k) \text { is a free rise of } x\right\} .
$$

Theorem 3 implies that $x_{i}<y_{i}$. Because $\left(i, x_{y_{i}}^{-1}\right)$ is a rise of $x$, there exists at least one element in the set of which $j$ is the minimum. Denote $(i j) x(i j)$ by $z$. We show that $z=y$ and $\operatorname{inv}(z)=\operatorname{inv}(x)+2$.

If $\left(x_{i}, x_{j}\right)$ is not a free rise of $x(i j)$, then there is some $k \in[n]$ such that $x_{i}<k<x_{j}$ and $x(i j)_{x_{i}}<x(i j)_{k}<x(i j)_{x_{j}}$, i.e., $i<x_{k}<j$. This contradicts $(i, j)$ being a free rise of $x$, thus $\left(x_{i}, x_{j}\right)$ is a free rise of $x(i j)$. Moreover, $\operatorname{inv}(z)=\operatorname{inv}(x)+2$ by Lemma 10 and $x<z$ by Theorem 4 . Now, note that $x_{k}=z_{k}$ for all $k \neq i, j, x_{i}, x_{j}$, and the following inequalities hold by definition: $z_{i}=x_{j} \leqslant y_{i} ; z_{j}=x_{i} \leqslant y_{j} ; z_{x_{i}}=j \leqslant y_{x_{i}} ; z_{x_{j}}=i \leqslant y_{x_{j}}$. Thus, $z[h, k] \leqslant y[h, k]$ for all $h, k \in[2 n]$, and Theorem 3 implies that $z \leqslant y$. By the assumption that $x \triangleleft y$, we have $y=z$, thus completing the proof.

\section{The structure of Bruhat order on $F_{2 n}$}

A few structural results concerning $F_{2 n}$ are obtained almost for free. In particular, the following result follows from Propositions 9 and 11.

Proposition 12. The poset $F_{2 n}$ is graded and ranked by the weight function wt.

Additionally, the minimal element in $F_{2 n}$ is $(12)(34) \cdots(2 n-1,2 n)$, and the maximal element is $(1,2 n)(2,2 n-1) \cdots(n, n-1)$. It was shown in [4] that the rank-generating function with respect to the weight function is $[1]_{q}[3]_{q} \cdots[2 n-1]_{q}$. Figure 5 shows the Hasse diagram of $F_{6}$, whose elements are represented in canonical cycle notation. Example 13 describes how $F_{2 n}$ is embedded in $S_{2 n}$ for the $n=2$ case. 


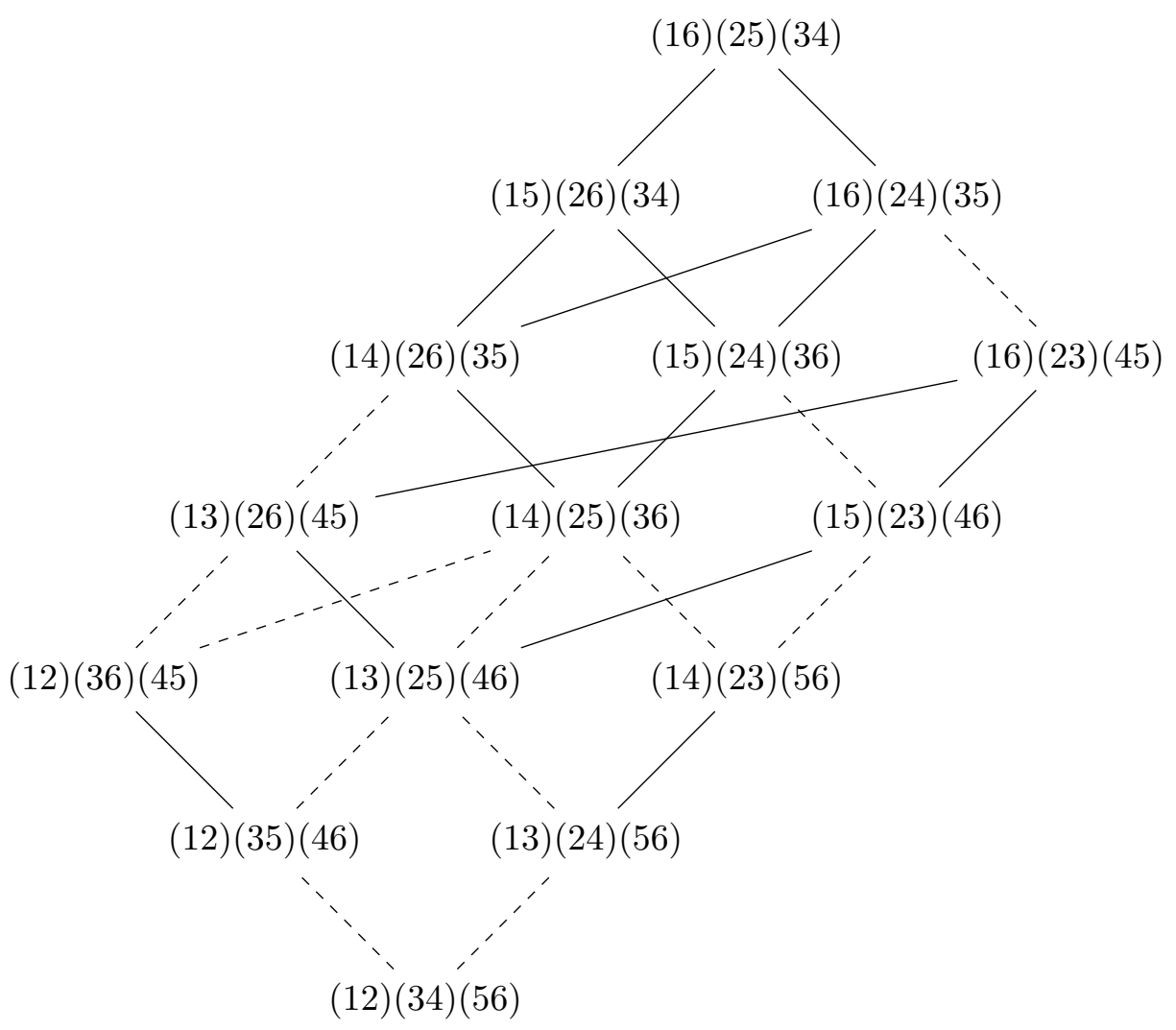

Figure 5: Bruhat order on $F_{6}$

Example 13. The poset $F_{4}$ corresponds to the union $[2134,3412] \cup[3412,4321]$ of intervals in $S_{4}$, which are pictured in Figure 6 . The three boldface elements comprise $F_{4}$, and the solid black diamonds correspond to the cover relations in $F_{4}$.

The remainder of this section details a structural decomposition of Bruhat order on $F_{2 n}$ which arises naturally when we realize fixed-point-free involutions as certain rook placements on Ferrers boards. We first proceed by recalling these combinatorial objects.

\subsection{Combinatorial tools}

\subsubsection{Dyck boards}

A board is a subset of $[n] \times[n]$ for some $n \in \mathbb{N}$, which we visualize as a subset of the cells on an $n \times n$ chessboard. We have already seen an example of a board: the permutation diagram of $\sigma \in S_{n}$ is the board $B(\sigma):=\left\{\left(i, \sigma_{i}\right): i \in[n]\right\} \subset[n] \times[n]$. A board $B$ is a Ferrers board if there exists a non-decreasing sequence $\left(b_{1}, b_{2}, \ldots, b_{n}\right)$ of positive integers, called the shape of $B$, such that $B=\left\{(i, j): i \in[n], j \in\left[b_{i}\right]\right\}$. A Ferrers board consists of $n$ adjacent columns of cells that share a bottom edge and whose heights $b_{i}$ are nondecreasing from left to right. 


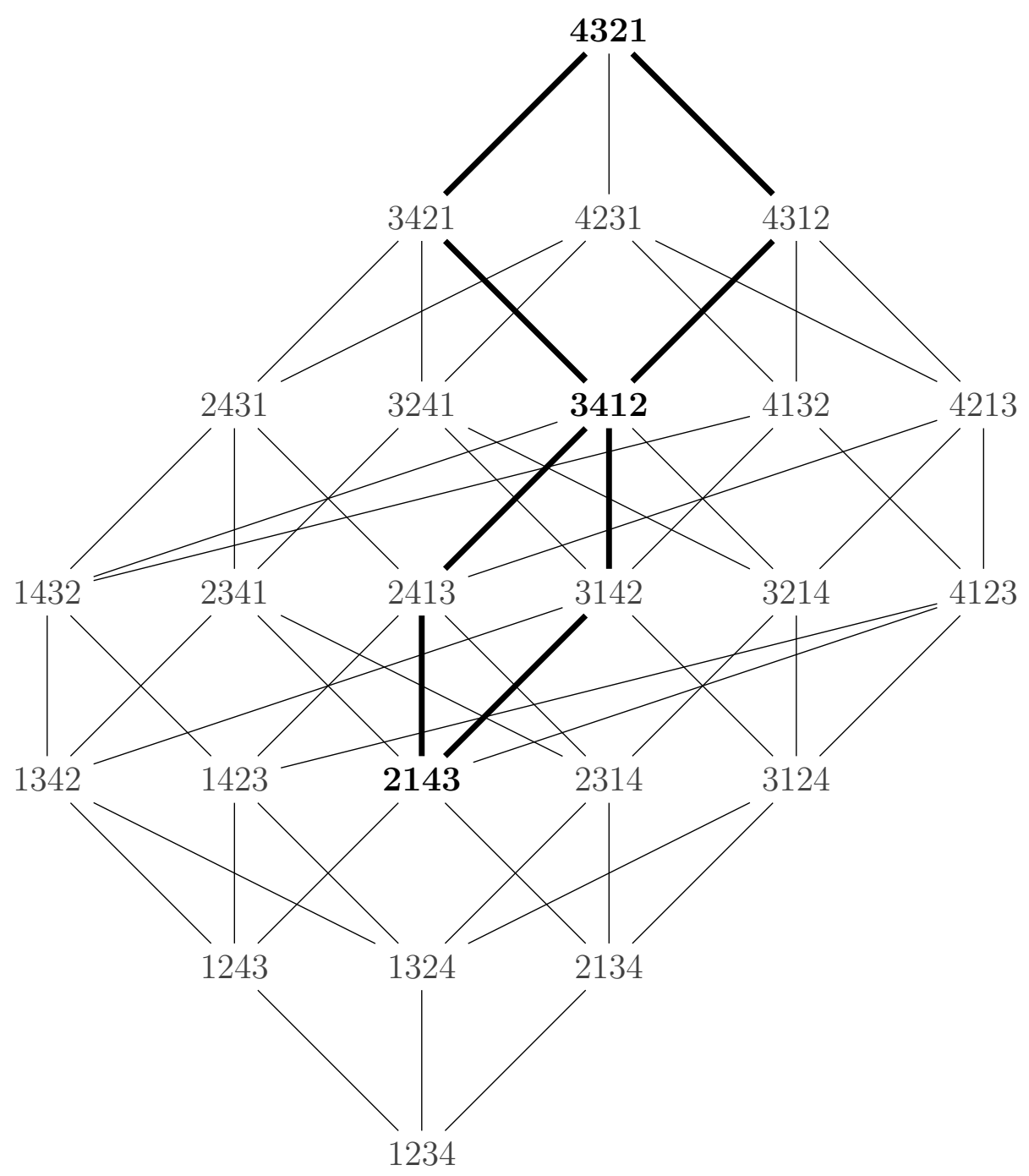

Figure 6: $F_{4}$, as an induced subposet of $S_{4}$

A Dyck word of length $2 n$ is a string $\delta$, consisting of $n$ es and $n d$ s such that no initial segment of $\delta$ contains more $d$ s than es. We denote the set of all Dyck words of length $2 n$ by $\mathcal{D}_{n}$. It is well-known that the Catalan numbers count Dyck words [13, Cor. 6.2.3].

For $\delta \in \mathcal{D}_{n}$, let $e_{i}(\delta)$ and $d_{i}(\delta)$ denote the positions in $\delta$ of the $i$ th $e$ and $i$ th $d$, respectively, for all $i \in[n]$. By letting an $e$ in $\delta$ represent a step $(0,1)$ to the north and a $d$ represent a step $(1,0)$ to the east, each Dyck word uniquely defines a Dyck path, which is a lattice path from $(0,0)$ to $(n, n)$, lying above the the main diagonal $\{(i, i): i \in[n]\}$ of cells.

Definition 14. The Dyck board $B(\delta)$ is the Ferrers board in $[n] \times[n]$ lying beneath the Dyck path $\delta \in \mathcal{D}_{n}$. That is, $B(\delta)$ is the Ferrers board with shape $\left(b_{1}, b_{2}, \ldots, b_{n}\right)$ where $b_{i}=d_{i}(\delta)-i$.

Example 15. Figure 7 shows the Dyck path of $\delta=$ eededded $\in \mathcal{D}_{4}$ and the corresponding 
Dyck board $B(\delta)$.

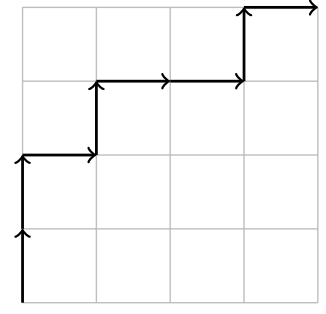

$\delta=e$ ededded

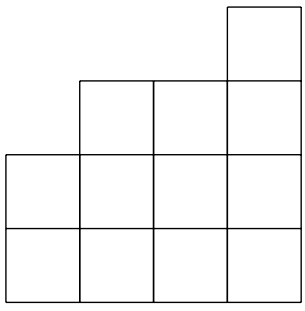

$B(\delta)$

Figure 7

Proposition 16. Suppose $\delta \in \mathcal{D}_{n}$. Then $(i, j) \in B(\delta)$ if and only if $e_{j}(\delta)<i+j$ if and only if $i+j \leqslant d_{i}(\delta)$.

Proof. The cell $(i, j)$ is contained in $B(\delta)$ if and only if the number of steps north occuring before the $i$ th step east is at least $j$. Additionally, the cell $(i, j)$ is contained in $B(\delta)$ if and only if the number of steps east occuring before the $j$ th step north is less than $i$.

We impose on $\mathcal{D}_{n}$ the following natural partial order: $\delta \leqslant_{\mathcal{D}} \delta^{\prime}$ if and only if $B(\delta) \subset$ $B\left(\delta^{\prime}\right)$. It is well-known that $\left(D_{n}, \leqslant \mathcal{D}\right)$ is a distributive lattice, which follows from viewing $\mathcal{D}_{n}$ as containment order on the set of order ideals of a certain poset. The rank function $\rho_{\mathcal{D}}$ of $\mathcal{D}_{n}$ is given by $\rho_{\mathcal{D}}(\delta)=\sum_{i \in[n]}\left(d_{i}(\delta)-2 i\right)$ for all $\delta \in \mathcal{D}_{n}$, and the rank of $\mathcal{D}_{n}$ is $\left(\begin{array}{l}n \\ 2\end{array}\right)$. In terms of the corresponding Dyck boards, the rank of $\delta$ can be interpreted as the number of cells of $B(\delta)$ lying strictly above the main diagonal, so that $\delta \triangleleft_{\mathcal{D}} \delta^{\prime}$ if and only if $B\left(\delta^{\prime}\right)$ is obtained by adding a cell to $B(\delta)$. Let $\mathcal{D}_{n}$ denote both the set of Dyck words of length $2 n$ and the poset $\left(\mathcal{D}_{n}, \leqslant_{\mathcal{D}}\right)$, as no confusion should arise. Figure 8 shows the Hasse diagrams of $\mathcal{D}_{3}$ and $\mathcal{D}_{4}$, with the elements represented by their corresponding Dyck boards.

\subsubsection{Rook placements}

A (non-attacking) $k$-rook placement on the board $B$ is a $k$-subset $P \subset B$ such that no two elements of $P$ lie in the same row or column. Let $r_{k}(B)$ denote the number of $k$-rook placements on the board $B$. Every $\sigma \in S_{n}$ corresponds to an $n$-rook placement on the board $[n] \times[n]$, thus $r_{n}(B)$ counts the number of permutations $\sigma \in S_{n}$ such that $B$ contains $B(\sigma)$. A Ferrers board $B$ with shape $\left(b_{1}, b_{2}, \ldots, b_{n}\right)$ contains $r_{n}(B)=\prod_{i=1}^{n}\left(b_{i}-i+1\right)$ $n$-rook placements, thus for a Dyck path $\delta \in \mathcal{D}_{n}$, we have

$$
r_{n}(B(\delta))=\prod_{i=1}^{n}\left(d_{i}(\delta)-2 i+1\right) .
$$

Example 17. Upon revisiting Figure 7, one finds that for $\delta=$ eededded, we have

$$
r_{0}(B(\delta))=1, r_{1}(B(\delta))=12, r_{2}(B(\delta))=38, r_{3}(B(\delta))=32, \text { and } r_{4}(B(\delta))=4
$$




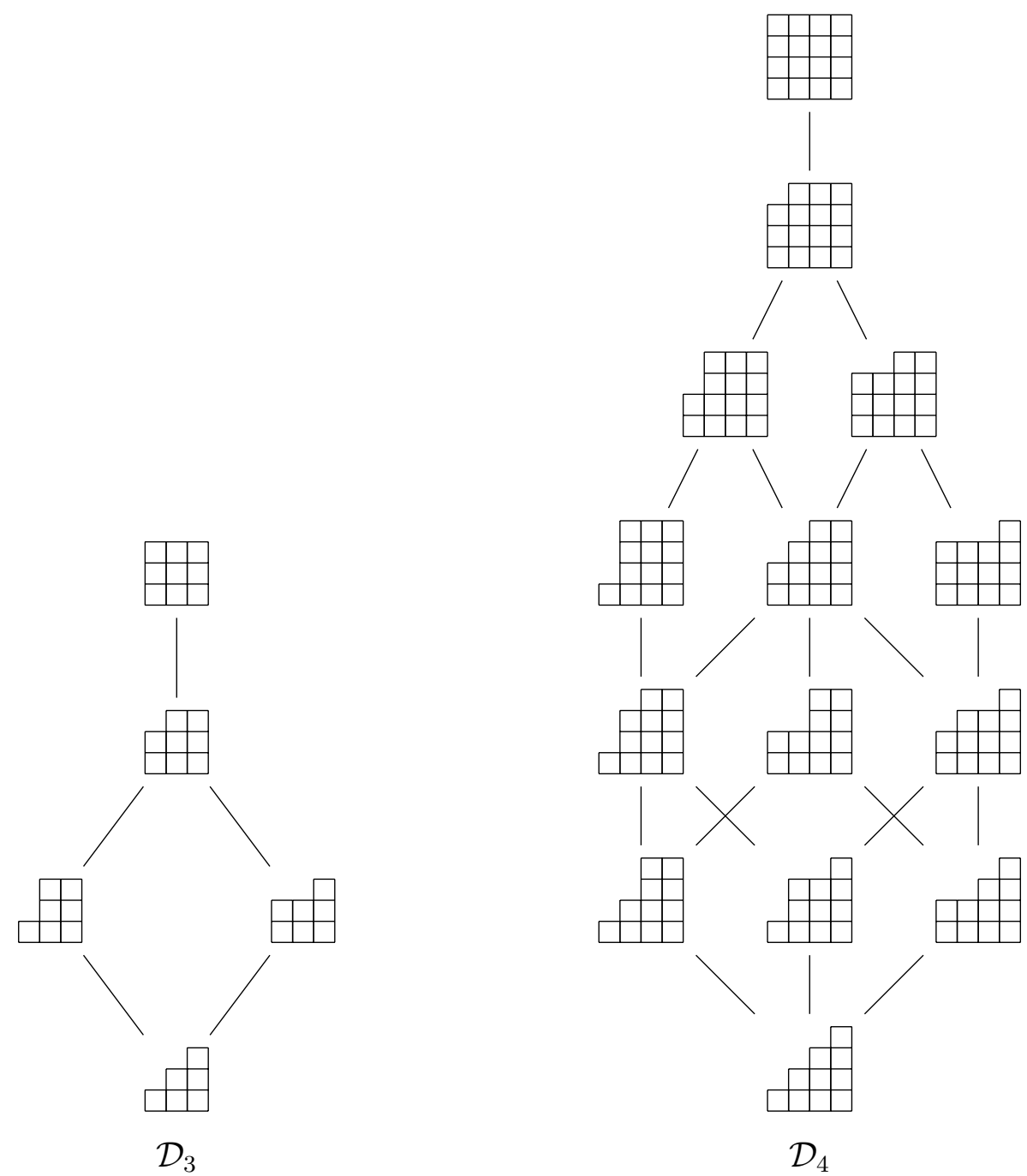

Figure 8

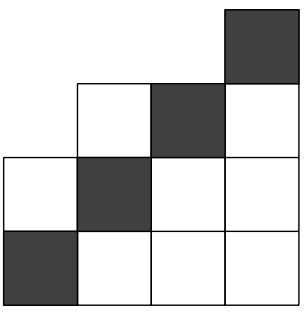

1234

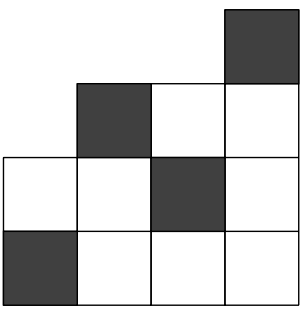

1324

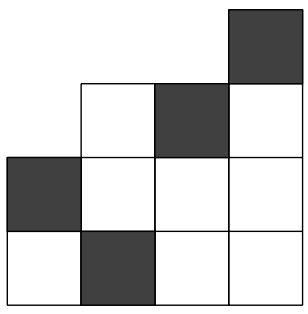

2134

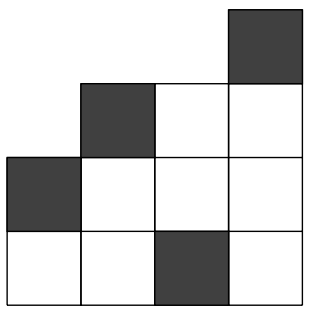

2314

Figure 9: The four 4-rook placements on B(eededded)

by inspection. The four rook placements on $B(\delta)$ are shown in Figure 9 .

There is an intimate order-theoretic relationship between Dyck paths and rook placements. In fact, Sjöstrand describes in [12, Corollary 5] that the elements in the lower 
Bruhat interval $\left[i d_{n}, \sigma\right]$ correspond to the number of rook placements on the the smallest right-aligned Ferrers matrix covering $B(\sigma)$. These Ferrers matrices are essentially rotations of our Ferrers boards, thus any Dyck path $\delta \in \mathcal{D}_{n}$ defines a unique order ideal of $S_{n}$ consisting of all permuations $\sigma \in S_{n}$ such that $B(\delta)$ contains $B(\sigma)$. This order ideal is principally generated by the permutation $\mu(\delta)$, where $\mu(\delta)_{i}:=\max \left(\left[d_{i}(\delta)-i\right] \backslash\left\{\mu(\delta)_{j}\right.\right.$ : $1 \leqslant j<i\}$ ). One can visually construct $\mu(\delta)$ on $B(\delta)$ by placing a rook as high as possible in each column from left-to-right while maintaining that no two rooks lie in the same row.

Example 18. Figure 10 depicts the board $B(\delta)$ for $\delta=$ eeeddedededd $\in \mathcal{D}_{6}$ on which the permutation diagram of $\mu(\delta)$ is superimposed.

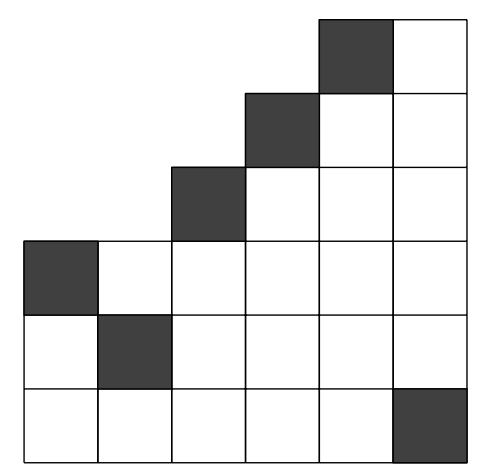

Figure 10: $\mu($ eeeddedededd $)=324561$

Proposition 19. For all $\delta \in \mathcal{D}_{n}$, the set of n-rook placements on $B(\delta)$ equals the set of permutations in the order ideal of $S_{n}$ generated by $\mu(\delta)$.

This proposition follows directly from the following two lemmas and noting that $B(\mu(\delta)) \subset B(\delta)$ by definition.

Lemma 20. Suppose $\delta \in \mathcal{D}_{n}$ and $\sigma \in S_{n}$. If $B(\sigma) \subset B(\delta)$, then $\sigma \leqslant_{B} \mu(\delta)$.

Proof. By definition, the value $\mu(\delta)_{i}$ is chosen maximally for each $i \in[n]$, thus $\mu(\delta)[h, k] \geqslant$ $\sigma[h, k]$ for all $h, k \in[n]$. Thus the result follows from Theorem 3 .

Lemma 21. Suppose $\delta \in \mathcal{D}_{n}$ and $\sigma \in S_{n}$. If $B(\sigma) \subset B(\delta)$ and $\tau \leqslant B$, then $B(\tau) \subset B(\delta)$.

Proof. Note that if a Ferrers board contains the cell $(i, j)$, then it contains the cells below and to its right. In other words, the Ferrers board contains the cell $(i, k)$ for all $1 \leqslant k \leqslant j$ and the cell $(l, j)$ for all $i \leqslant l \leqslant n$. Now, if $\tau \triangleleft_{B} \sigma$, then $\tau(i j)=\sigma$ for some free rise $(i, j)$ of $\tau$. Then $i<j$ and $\tau_{i}=\sigma_{j}<\sigma_{i}=\tau_{j}$, and $B(\delta)$ contains the cells $\left(i, \tau_{j}\right)$ and $\left(j, \tau_{i}\right)$. Thus $B(\tau) \subset B(\delta)$.

In addition to this fact, the following proposition details why $\mu$ is of order-theoretic interest. The result follows from the three lemmas that follow it.

Proposition 22. The map $\mu$ is an order-isomorphism from $\mathcal{D}_{n}$ to $S_{n}(312)$ under Bruhat order. 
Lemma 23. For any $\delta \in \mathcal{D}_{n}$, the permutation $\mu(\delta)$ is 312-avoiding.

Proof. Suppose $\delta \in \mathcal{D}_{n}$. The definition of $\mu$ ensures that the value $\mu(\delta)_{i}$ is chosen to be the maximum possible value not already appearing in $\mu(\delta)$ before the $i$ th position, so $\mu(\delta)$ never has $\mu(\delta)_{j}<\mu(\delta)_{k}<\mu(\delta)_{i}$ for any triple $i<j<k$. Thus $\mu\left(\mathcal{D}_{n}\right) \subseteq S_{n}(312)$.

Lemma 24. The map $\mu$ is a bijection from $\mathcal{D}_{n}$ to $S_{n}(312)$

Proof. We already have $\mu\left(\mathcal{D}_{n}\right) \subseteq S_{n}(312)$ by Lemma 22. Thus it suffices to show $\mu$ is injective because $\mathcal{D}_{n}$ and $S_{n}(312)$ have the same cardinality. Suppose $\delta \neq \delta^{\prime} \in \mathcal{D}_{n}$, and let $i>1$ be the least index such that $\delta_{i} \neq \delta_{i}^{\prime}$. Without loss of generality, suppose $\delta_{i}=d$ and $\delta_{i}^{\prime}=e$. Since it has been assumed that $\delta_{j}=\delta_{j}^{\prime}$ for all $1 \leqslant j<i$, we have $\mu(\delta)_{j}=\mu\left(\delta^{\prime}\right)_{j}$ for all $1 \leqslant j<i$, thus

$$
\begin{aligned}
\mu(\delta)_{i} & =\max \left(\left[d_{i}(\delta)-i\right] \backslash\left\{\mu(\delta)_{j}: 1 \leqslant j<i\right\}\right) \\
& <\max \left(\left[d_{i}\left(\delta^{\prime}\right)-i\right] \backslash\left\{\mu\left(\delta^{\prime}\right)_{j}: 1 \leqslant j<i\right\}\right)=\mu\left(\delta^{\prime}\right)_{i} .
\end{aligned}
$$

Thus, $\mu(\delta)_{i} \neq \mu\left(\delta^{\prime}\right)_{i}$, so $\mu(\delta) \neq \mu\left(\delta^{\prime}\right)$, and we have proved that $\mu$ is injective.

Lemma 25. $\delta \leqslant_{\mathcal{D}} \delta^{\prime}$ if and only if $\mu(\delta) \leqslant_{B} \mu\left(\delta^{\prime}\right)$.

Proof. Suppose $\delta \leqslant \mathcal{D} \delta^{\prime}$. By definition, then, $d_{i}(\delta)-i \leqslant d_{i}(\delta)-i$ for all $i \in[n]$, and thus $\max \left(\left[d_{i}(\delta)-i\right]\right) \leqslant \max \left(\left[d_{i}\left(\delta^{\prime}\right)-i\right]\right)$ for all $i \in[n]$, so $\mu(\delta) \leqslant B \mu(\delta)$. Conversely, suppose $\mu(\delta) \leqslant_{B} \mu\left(\delta^{\prime}\right)$. For all $i \in[n]$, define $j(i):=\max \left(\left\{\mu(\delta)_{k}: k \in[i]\right\}\right)$. Then $(i, j(i))=d_{i}(\delta)-i$ and $(i, j(i)) \in B\left(\delta^{\prime}\right)$ for all $i \in[n]$. Thus $B(\delta) \subset B\left(\delta^{\prime}\right)$, and $\delta \leqslant_{\mathcal{D}} \delta^{\prime}$.

\subsubsection{Hooks and hook lengths}

For the Dyck path $\delta$, define the reduced hook $H_{\delta}(i, j)$ of cell $(i, j) \in B(\delta)$ to be the union of the cells in $B(\delta)$ in the $j$ th row strictly to the left of cell $(i, j)$ and those in the $i$ th column strictly above cell $(i, j)$. Define the reduced hook length $h_{\delta}(i, j)$ of cell $(i, j) \in B(\delta)$ to be the cardinality of $H_{\delta}(i, j)$. (The hook length, usually seen in the context of Young tableaux, counts the cell $(i, j)$. It benefits us here to disregard it, hence the adjective reduced.) The following result shows that one can compute hook lengths on $B(\delta)$ directly from the Dyck word $\delta$.

Proposition 26. For all $\delta \in \mathcal{D}_{n}$, the reduced hook length of the cell $(i, j) \in B(\delta)$ is $h_{\delta}(i, j)=d_{i}(\delta)-e_{j}(\delta)-1$.

Proof. There are $d_{i}(\delta)-i$ cells in the $i$ th column of $B(\delta)$, and $d_{i}(\delta)-i-j$ of them lie above cell $(i, j)$. Similarly, the $j$ th row of $B(\delta)$ contains $n-\left(e_{j}(\delta)-j\right)$ cells, and $i+j-e_{j}(\delta)-1$ of them lie to the left of cell $(i, j)$.

Example 27. For $\delta=$ eededded, we have $h_{\delta}(3,2)=d_{3}(\delta)-e_{2}(\delta)-1=3$, as illustrated by Figure 11, in which the corresponding reduced hook $h_{\delta}(3,2)$ is shaded. 


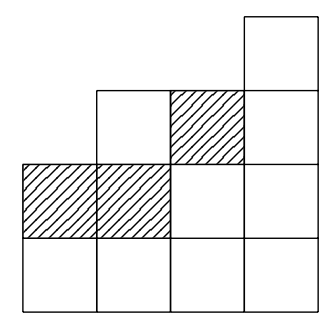

Figure 11: $h_{\delta}(3,2)=3$ for $\delta=$ eededded.

For all $\sigma \in S_{n}$ and $\delta \in \mathcal{D}_{n}$ such that $B(\sigma) \subset B(\delta)$, define the hook sum of the pair $(\delta, \sigma)$ to be $h(\delta, \sigma):=\sum_{i \in[n]} h_{\delta}\left(i, \sigma_{i}\right)$. If the board $B(\delta)$ contains the rook placements $B(\sigma)$ and $B(\tau)$ for two permutations $\sigma, \tau \in S_{n}$, then $\left\{e_{\sigma_{i}}(\delta)\right\}_{i=1}^{n}=\left\{e_{\tau_{i}}(\delta)\right\}_{i=1}^{n}$ and $\left\{d_{\sigma_{i}}(\delta)\right\}_{i=1}^{n}=$ $\left\{d_{\tau_{i}}(\delta)\right\}_{i=1}^{n}$. Thus appealing to Proposition 26 and rearranging the summands in the hook sums justifies the following result.

Proposition 28. For any $\delta \in \mathcal{D}_{n}$, all n-rook placements on the Dyck board $B(\delta)$ have equal hook sums.

Because $h(\delta, \sigma)$ does not depend on the choice of $\sigma$, we define $h(\delta):=h\left(\delta, i d_{n}\right)$ for all $\delta \in \mathcal{D}_{n}$. The cells in $B(\delta)$ strictly above the main diagonal are counted exactly twice by $h(\delta)$, implying that the rank of any $\delta \in \mathcal{D}_{n}$ is $h(\delta) / 2$.

Define the crossing number $c(\delta, \sigma)$ to be the number of pairs of hooks that intersect. It is plain to see that if $i<j$ and the two hooks $H_{\delta}\left(i, \sigma_{i}\right)$ and $H_{\delta}\left(j, \sigma_{j}\right)$ intersect, then $(i, j)$ is a rise of $\sigma$ and $\left(i, \sigma_{j}\right)$ is the cell in the intersection, provided that it lies in $B(\delta)$.

Proposition 29. Suppose $\delta \in \mathcal{D}_{n}$ and $\sigma \in S_{n}$ such that $B(\sigma) \subset B(\delta)$. Then the two hooks $H_{\delta}\left(i, \sigma_{i}\right)$ and $H_{\delta}\left(j, \sigma_{j}\right)$ intersect at $\left(i, \sigma_{j}\right)$ if and only if $\left(i, \sigma_{j}\right) \in B(\delta)$.

As we see next, the map $\mu$ yields the unique rook placement on a given Dyck board with disjoint hooks.

Proposition 30. If $\delta \in \mathcal{D}_{n}$ and $\sigma \in S_{n}$, then $c(\delta, \sigma)=0$ if and only if $\sigma=\mu(\delta)$.

Proof. Suppose $\delta \in \mathcal{D}_{n}$ and $\sigma \in S_{n}$. We have $c(\delta, \sigma) \neq 0$ if and only if there exist values $1 \leqslant i<j \leqslant n$ such that $\sigma_{i}<\sigma_{j}$ and $H_{\delta}\left(i, \sigma_{i}\right) \cap H_{\delta}\left(j, \sigma_{j}\right)=\left\{\left(i, \sigma_{j}\right)\right\}$, i.e., $\sigma_{i}<\sigma_{j} \leqslant d_{i}-i$. This occurs if and only if $\sigma \neq \mu(\delta)$.

Example 31. Consider $\delta=$ eededded $\in \mathcal{D}_{4}$, as in Figures 9 and 11. By inspection, one can verify the following: each $\sigma \in S_{4}$ with $B(\sigma) \subset B(\delta)$ has $h(\delta, \sigma)=4$; the corresponding crossing numbers are $c(\delta, 1234)=2, c(\delta, 1324)=1, c(\delta, 2134)=1$, and $c(\delta, 2314)=0$; $\mu(\delta)=2314$; and the rank of $\delta$ is $\rho_{\mathcal{D}}(\delta)=2$.

\section{$3.2 \quad F_{2 n}$ as rook placements on Dyck boards}

Recall that the edges in the arc diagram of $x \in F_{2 n}$ are represented in the canonical cycle notation by disjoint ordered pairs $(e, d) \in[n]^{2}$ such that $x_{d}=e<d=x_{e}$, and note that 
any $(e d) \in x$ defines an arc whose terminal point $d$ is paired with the earlier-appearing initial point $e$. Therefore the arrangement of exceedances and deficiencies, as they occur in the one-line notation of $x \in F_{2 n}$, forms a Dyck word in $\mathcal{D}_{n}$.

Definition 32. For $x \in F_{2 n}$, define $\delta(x)$ to be the Dyck word $\delta(x)_{1} \delta(x)_{2} \cdots \delta(x)_{2 n}$ with

$$
\delta(x)_{i}:=\left\{\begin{array}{lll}
e & : & x_{i}>i \\
d & : & x_{i}<i
\end{array}\right.
$$

The remaining characteristic that uniquely determines a fixed-point-free involution is the order in which its $n$ arcs terminate.

Definition 33. For $x \in F_{2 n}$, define $\sigma(x)$ to be the permutation with $\sigma(x)_{i}=j$ if and only if the arc diagram of $x$ has an arc from $e_{j}(\delta(x))$ to $d_{i}(\delta(x))$ for all $i \in[n]$.

For any $x \in F_{2 n}$, the permutation $\sigma(x)$ can be constructed easily on the arc diagram of $x$ by indexing the es and $d \mathrm{~s}$ of $\delta(x)$ in the order they appear with the set $[n]$ and tracing the arcs backward to realize $\sigma(x)$ as the bijection from the $d$-index set to the $e$-index set.

Example 34. Consider the fixed-point-free involution $x=73286514$ in Figure 4 . Then $\delta(x)=$ eedeeddd and $\sigma(x)=2413$. Figure 12 illustrates the construction detailed in the preceding paragraph.

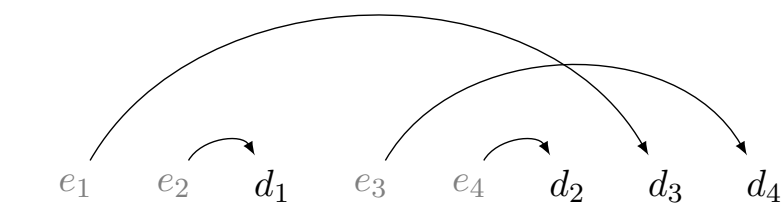

Figure 12: For $x=73286514 \in F_{8}$, $\delta(x)=$ eedeeddd $\in \mathcal{D}_{4}$ and $\sigma(x)=2413 \in S_{4}$.

It should be noted that we could equivalently regard $\sigma$ as a map from the $e$-index set to the $d$-index set. This may seem more natural considering the direction of the arrows, and in this case, $\sigma$ would map to the inverse permutation. However, our definition of $\sigma$ is more natural in the sense that it corresponds to rook placements under Dyck paths consisting of north and east steps and thus on Ferrers boards that are bottom- and right-justified.

Definition 35. Define the map $\varphi: F_{2 n} \rightarrow \mathcal{D}_{n} \times S_{n}$ by $\varphi(x)=(\delta(x), \sigma(x))$.

Proposition 36. The map $\varphi$ is a bijection from $F_{2 n}$ to the set

$$
\left\{(\delta, \sigma) \in \mathcal{D}_{n} \times S_{n}: B(\sigma) \subset B(\delta)\right\} .
$$


Proof. To prove $\varphi$ is surjective, suppose $(\delta, \sigma) \in\left\{(\delta, \sigma) \in \mathcal{D}_{n} \times S_{n}: B(\sigma) \subset B(\delta)\right\}$. Then $\sigma_{i} \leqslant d_{i}(\delta)-i$ for all $i \in[n]$. These inequalities imply the existence of a valid arc diagram, and thus a fixed-point-free involution $x=\left(e_{\sigma_{1}}, d_{1}\right)\left(e_{\sigma_{2}}, d_{2}\right) \cdots\left(e_{\sigma_{n}}, d_{n}\right)$ in $F_{2 n}$ such that $\varphi(x)=(\delta, \sigma)$. As for the injectivity of $\varphi$, suppose $\varphi(x)=\varphi(y)$ so that $\delta(x)=\delta(y)$ and $\sigma(x)=\sigma(y)$. Then the transpositions $\left(e_{\sigma(x)_{i}}(\delta(x)), d_{i}(\delta(x))\right)$ appear in the canonical cycle notation for both $x$ and $y$ for every $i \in[n]$. Thus $x=y$, and the proof is complete.

Example 37. The map $\varphi$ identifies the four rook placements on Dyck boards shown in Figure 9 with the four fixed-point-free involutions 35162487, 36154287, 53261487, and 63254187 , respectively.

Proposition 38. The map $\delta: F_{2 n} \rightarrow \mathcal{D}_{n}$ is order-preserving.

Proof. Suppose $x \triangleleft y$ in $F_{2 n}$. Then by Proposition 11, there is a free rise $(i, j)$ of $x$ such that $y=(i j) x(i j)=\left(x_{i} x_{j}\right) x\left(x_{i} x_{j}\right)$. Thus

$$
y_{i}=x_{j}, \quad y_{j}=x_{i}, \quad y_{x_{i}}=j, \quad \text { and } \quad y_{x_{j}}=i .
$$

To prove $\delta(x) \leqslant_{\mathcal{D}} \delta(y)$, it suffices to consider positions $i, j, x_{i}$, and $x_{j}$ because all other positions have equal values. The definition of a rise guarantees $i<j$ and $x_{i}<x_{j}$, and we can further require $i<x_{i}$ by swapping $i$ and $x_{i}$ if necessary. There are three cases to consider: (1) $i<j<x_{i}<x_{j}$, (2) $i<x_{i}<x_{j}<j$, and (3) $i<x_{i}<j<x_{j}$.

In the first case, $\delta(x)_{i}=e, \delta(x)_{j}=e, \delta(x)_{x_{i}}=d$, and $\delta(x)_{x_{j}}=d$.

$$
\begin{aligned}
y_{i}=x_{j}>i & \Rightarrow \delta(y)_{i}=e \\
y_{j}=x_{i}>j & \Rightarrow \delta(y)_{j}=e \\
y_{x_{i}}=j<x_{i} & \Rightarrow \delta(y)_{x_{i}}=d \\
y_{x_{j}}=i<x_{j} & \Rightarrow \delta(y)_{x_{j}}=d
\end{aligned}
$$

In the second case, $\delta(x)_{i}=e, \delta(x)_{x_{i}}=d, \delta(x)_{x_{j}}=e$, and $\delta(x)_{j}=d$.

$$
\begin{aligned}
y_{i}=x_{j}>i & \Rightarrow \delta(y)_{i}=e \\
y_{x_{i}}=j>x_{i} & \Rightarrow \delta(y)_{x_{i}}=e \\
y_{x_{j}}=i<x_{j} & \Rightarrow \delta(y)_{x_{j}}=d \\
y_{j}=x_{i}<j & \Rightarrow \delta(y)_{j}=d
\end{aligned}
$$

The third case would imply that $x(i j)$ is not covered by $y$ in Bruhat order on $S_{2 n}$ because $\left(x_{i}, x_{j}\right)$ would not be a free rise of $x(i j)$, contradicting the assumption that $x \triangleleft y$.

Thus the subsequence of the Dyck word $\delta(x)$ affected by moving up in $F_{2 n}$ via a cover relation is either eedd or eded, and in both cases, $\delta(y)_{i}=\delta(y)_{j}=e$ and $\delta(y)_{x_{i}}=\delta(y)_{x_{j}}=$ $d$. In other words, the affected subsequence becomes eedd in $\delta(y)$, while the remainder of the two Dyck words are equal. Thus $\delta(x) \leqslant \mathcal{D} \delta(y)$. 


\subsection{Structural decomposition}

Recall that Proposition 12 asserts that the Bruhat order on $F_{2 n}$ has rank function wt $(x)=$ $\operatorname{span}(x)-\operatorname{cross}(x)$. We have developed the necessary tools to restate this function in terms of our representation of elements of $F_{2 n}$ as rook placements on Dyck boards.

Proposition 39. If $x \in F_{2 n}$, then $\operatorname{span}(x)=(h \circ \varphi)(x)$ and $\operatorname{cross}(x)=(c \circ \varphi)(x)$.

Proof. The first equality is immediate from the definition of span, the definition of hook length, and Proposition 26.Propositions 8, 16, and 29 imply that arcs in the arc diagram of a fixed-point-free involution intersect if and only if the corresponding hooks intersect on its Dyck board, thus justifying the second equality.

In order to prove Theorem 1, we reveal the structural properties of Bruhat order on $F_{2 n}$ with respect to two equivalence relations $\Delta$ and $\Sigma$, which we define now. For $x, y \in F_{2 n}$, define $x \equiv_{\Delta} y$ if and only if $\delta(x)=\delta(y)$, and define $x \equiv_{\Sigma} y$ if and only if $\sigma(x)=\sigma(y)$. We denote the equivalence classes of $x$ under $\Delta$ and $\Sigma$ by $[x]_{\Delta}$ and $[x]_{\Sigma}$, respectively, and we consider these equivalence classes as induced subposets of $F_{2 n}$.

Propositions 19 and 36 imply that the map $\sigma$ restricts to a bijection between $[x]_{\Delta}$ and the order ideal of $S_{n}$ generated by the 312-avoiding permutation $\mu(\delta(x))$. However, a stronger fact holds: they are isomorphic posets.

Lemma 40. The map $\sigma$ restricts to an order-isomorphism from $[x]_{\Delta}$ to the principal order ideal of $S_{n}$ generated by $\mu(\delta(x))$.

Proof. Consider two fixed-point-free involutions $x, y \in F_{2 n}$ such that $y \in[x]_{\Delta}$. The map $\delta$ is order-preserving by Proposition 38 , and thus $[x]_{\Delta}$ is order-convex. The cover relation $x \triangleleft y$ exists in $F_{2 n}$ if and only if $y=(i j) x(i j)$ for a free rise $(i, j)$ of $x$ and $\operatorname{inv}(y)=\operatorname{inv}(x)+2$.

Choose $i$ and $j$ to be deficiencies (by swapping $i$ and $j$ for $x_{i}$ and $x_{j}$, respectively, if necessary), so that $d_{a}:=d_{a}(\delta(x))=i$ and $d_{b}:=d_{d}(\delta(x))=j$ for some $a, b$ with $a<b$.

This cover relation occurs in $F_{2 n}$ if and only if any arc terminating between $d_{a}$ and $d_{b}$ has its initial point between $x_{d_{a}}$ and $x_{d_{b}}$, i.e., between $e_{\sigma(x)_{a}}(\delta(x))$ and $e_{\sigma(x)_{b}}(\delta(x))$. In other words, $(a, b)$ is a free rise of $\sigma(x)$, and $\sigma(y)=\sigma(x)(a b)$. Thus $\sigma(x) \triangleleft_{B} \sigma(y)$.

Figure 13 illustrates the correspondence described in the proof of Proposition 40. An analogous fact about the map $\delta$ on $\Sigma$-classes is also true.

Lemma 41. For all $x \in F_{2 n}$, the map $\delta$ restricts to an order-isomorphism from $[x]_{\Sigma}$ to the induced subposet of $\mathcal{D}_{n}$ consisting of Dyck paths $\delta$ such that $B(\delta) \subset B(\sigma(x))$.

Proof. Proposition 36 implies that $\delta$ restricts to a bijection from $[x]_{\Sigma}$ to the set of Dyck paths containing $B(\sigma(x))$. For the remainder of the proof, consider two fixed-point-free involutions $x, y \in F_{2 n}$ such that $\varphi(x)=(\delta, \pi)$ and $\varphi(y)=\left(\delta^{\prime}, \pi\right)$, so that $y \in[x]_{\Sigma}$.

Suppose $\delta \triangleleft_{\mathcal{D}} \delta^{\prime}$ in $\mathcal{D}_{n}$, and we show $x \triangleleft y$ in $F_{2 n}$. Then $\delta$ and $\delta^{\prime}$ are identical in all positions except for some two adjacent positions $i$ and $i+1$, in which $\delta$ has de and $\delta^{\prime}$ has $e d$. Define $z:=(i i+1) x(i i+1)$, and the following two claims imply $z=y$. 


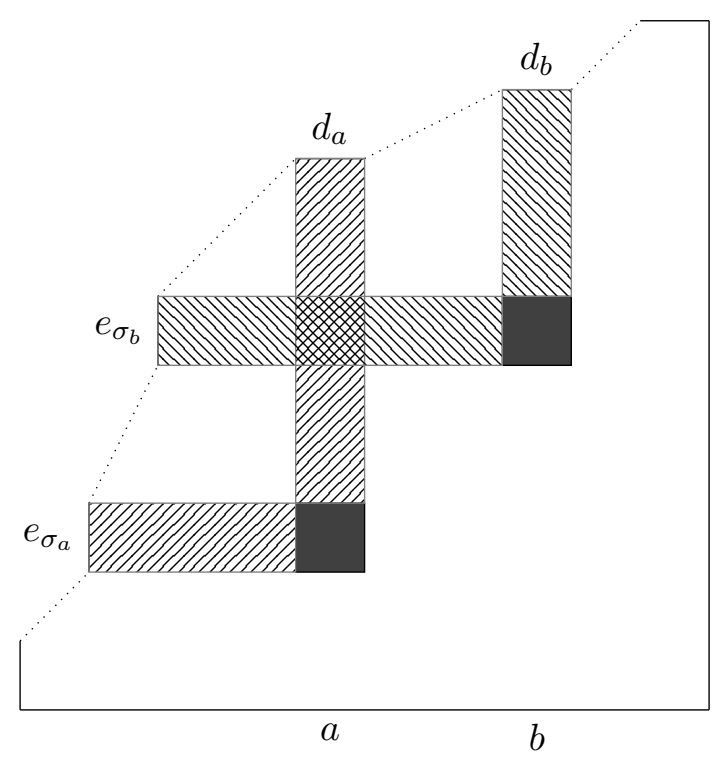

Figure 13

Claim 1: $\delta(z)=\delta^{\prime}$ : Note that $z_{i}=x_{i+1}>i+1>i$ and $z_{i+1}=x_{i}<i<i+1$, thus $\delta(z)_{i}=e$ and $\delta(z)_{i+1}=d$. Additionally, $z_{x_{i}}=i+1>i>x_{i}$ and $z_{x_{i+1}}=i<i+1<$ $x_{i+1}$, thus $\delta(z)_{x_{i}}=e$ and $\delta(z)_{x_{i+1}}=d$.

Claim 2: $z \in[x]_{\Sigma}$ : Aside from the $k$ th $d$ shifting one position to the right, we have $d_{m}(\delta(z))=d_{m}(\delta)$ for all $m \neq k$. Therefore the order of the $d$ s in does not change from $\delta$ to $\delta(z)$, and thus $\sigma(z)=\sigma(x)$.

To conclude that $x \triangleleft y$ in $F_{2 n}$, we note that the aforementioned $d e \mapsto e d$ swap from $\delta$ to $\delta^{\prime} \operatorname{implies} \operatorname{span}(y)=\operatorname{span}(x)+2$ and $\operatorname{cross}(y)=\operatorname{cross}(x)+1$. Thus $\operatorname{inv}(y)=\operatorname{inv}(x)+2$, by Proposition 9. Now, because $i$ and $i+1$ are adjacent positions, this $x_{i}$ and $x_{i+1}$ are adjacent in value. Thus it must be the case that $(i, i+1)$ is a free rise of $x$, and thus $x \triangleleft y$.

Now, to prove the converse, we suppose $x \triangleleft y$ in $F_{2 n}$. Then $y=(i j) x(i j)$ for a free rise $(i, j)$ of $x$ and $\operatorname{inv}(y)=\operatorname{inv}(x)+2$. It is either the case, then, that $i$ is a deficiency and $j$ is an exceedance or $i$ is an exceedance and $j$ is a deficiency. Choose $i$ and $j$ so that the former holds (by swapping $i$ and $j$ with $x_{i}$ and $x_{j}$, respectively, if the latter holds). Note that $j=i+k$, so that $\operatorname{cross}(y)=\operatorname{cross}(x)+k$. Because $\operatorname{inv}(y)=\operatorname{inv}(x)+2$, we must have $k=1$, and $\delta$ and $\delta^{\prime}$ are identical in all positions except $i$ and $i+1$ in which $\delta$ has de and $\delta^{\prime}$ has $e d$. Thus $\delta \triangleleft \delta^{\prime}$.

The remaining structural information about $F_{2 n}$ is how the equivalence classes under $\Delta$ are ordered in $F_{2 n}$, with respect to one another. We recall from [10] the definition of a poset congruence and the natural partial order on the quotient. An equivalence relation $\Theta$ on the elements of a poset $P$ is a poset congruence if

(i) every equivalence class $[x]_{\Theta}$ of $x \in P$ is an interval in the poset $P$, 
(ii) the projection $\Theta^{\uparrow}$ mapping $x$ to the maximal element in its equivalence class is order-preserving, and

(iii) the projection $\Theta^{\downarrow}$ mapping $x$ to the minimal element in its equivalence class is order-preserving.

Define a partial order on the congruence classes of $P$ under $\Theta$ by $[x]_{\Theta} \leqslant[y]_{\Theta}$ if and only if some $x^{\prime} \in[x]_{\Theta}$ and $y^{\prime} \in[y]_{\Theta}$ exist such that $x^{\prime} \leqslant_{P} y^{\prime}$. The quotient of $P$ with respect to the poset congruence $\Theta$, denoted $P / \Theta$, consists of the set of congruence classes under $\Theta$ under this partial order. The quotient $P / \Theta$ is isomorphic to the subposet $\Theta^{\downarrow}(P) \subset P$ of minimal representatives in each class. We identify these two isomorphic posets. We proceed by showing that the equivalence $\Delta$ satisfies this definition.

Proposition 42. The equivalence $\Delta$ is a poset congruence.

Proof. Each of the three claims below respectively address the three parts of the definition of a poset congruence detailed above.

Claim 1. The equivalence class $[x]_{\Delta} \in F_{2 n} / \Delta$ is an interval: This is immediate because $\delta$ is order-preserving and $[x]_{\Delta}$ is order-isomorphic to the order ideal in $S_{n}$ generated by $\mu(\delta(x))$ by Proposition 38 and Lemma 40, respectively. Thus for all $x \in F_{2 n}$,

(a) $\Delta^{\uparrow}(x)$ is the fixed-point-free involution $y \in[x]_{\Delta}$ with $\sigma(y)=\mu(\delta(x))$, and

(b) $\Delta^{\downarrow}(x)$ is the fixed-point-free involution $y \in[x]_{\Delta}$ with $\sigma(y)=i d_{n}$.

Claim 2. The projection $\Delta^{\uparrow}$ is order-preserving: Suppose $x \leqslant y$ in $F_{2 n}$. (In order to show $\Delta^{\uparrow}(x) \leqslant \Delta^{\uparrow}(y)$, we prove the existence of an element $z$ such that $\Delta^{\uparrow}(x) \leqslant$ $z \leqslant \Delta^{\uparrow}(y)$.) Because the maps $\delta: F_{2 n} \rightarrow \mathcal{D}_{n}$ and $\mu: \mathcal{D}_{n} \rightarrow S_{n}$ are order-preserving (by Propositions 38 and 22), the composition $\mu \circ \delta$ is also order-preserving, i.e., $\mu(\delta(x)) \leqslant_{B} \mu(\delta(y))$. By Lemma 40, the Dyck board $B(\delta(y))$ contains $B(\mu(\delta(x)))$, and Proposition 36 guarantees the existence of a fixed-point-free involution $z$ such that $\varphi(z)=(\delta(y), \mu(\delta(x)))$. Now, Lemma 40 implies that $\Delta^{\uparrow}(x) \leqslant z$ in $[z]_{\Sigma}$, and Lemma 41 implies $z \leqslant \Delta^{\uparrow}(y)$ in $[y]_{\Delta}$. Thus $\Delta^{\uparrow}(x) \leqslant z \leqslant \Delta^{\uparrow}(y)$.

Claim 3. The projection $\Delta^{\downarrow}$ is order-preserving: Again, consider $x, y \in F_{2 n}$ such that $x \leqslant y$. Note that $\varphi\left(\Delta^{\downarrow}(x)\right)=\left(\delta(x), i d_{n}\right)$ and $\varphi\left(\Delta^{\downarrow}(y)\right)=\left(\delta(y), i d_{n}\right)$. Recall $\delta(x) \leqslant \delta(y)$ by Lemma 41 . Thus $\Delta^{\downarrow}(x) \leqslant \Delta^{\downarrow}(y)$ by Lemma 40 .

Thus $\Delta$ is a poset congruence.

Proposition 43. The quotient $F_{2 n} / \Delta$ is isomorphic to the poset $\mathcal{D}_{n}$.

Proof. Note that $x \in \Delta^{\downarrow}\left(F_{2 n}\right)$ if and only if $\sigma(x)=i d_{n}$, Now, Lemma 41 says that $[x]_{\Sigma}$ is isomorphic to $\mathcal{D}_{n}$.

One can verify Proposition 43 for the $n=3$ case by comparing the the poset $\mathcal{D}_{3}$ in Figure 8 to the induced subposet $\Delta^{\downarrow}\left(F_{6}\right)$ in Figure 2. We now prove our main result. 
Proof of Theorem 1. By Lemmas 40 and 41 and the fact that $\Delta^{\downarrow}(x)=\left(\delta(x), i d_{n}\right)$, the rank function $\rho$ of $F_{2 n}$ can be expressed as the sum of the rank functions on $\mathcal{D}_{n}$ and $S_{n}$. Specifically, if $x \in F_{2 n}$ and $\varphi(x)=(\delta, \sigma)$, then $\rho(x)=\rho_{\mathcal{D}}(\delta)+\operatorname{inv}(\sigma)$.

Recall that the rank-generating function for $\mathcal{D}_{n}$ is $\sum_{i \in[n]} d_{i}(\delta)-2 i$, justifying the factors of the form $q^{d_{i}(\delta)-2 i}$ in the expansion. Also recall from Section 3.1.2 the rank-generating function of lower Bruhat intervals. Of concern here are the intervals $\left[i d_{n}, \mu(\delta)\right] \subseteq S_{n}$ for $\delta \in \mathcal{D}_{n}$. The rank-generating function for this interval is

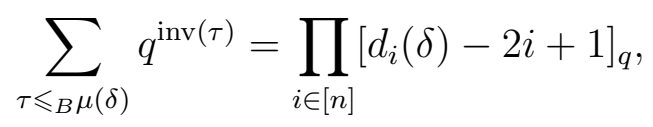

a fact that follows from Lemma 40. This justifies the remaining factors, and thus proves Theorem 1.

Now, setting $q=1$ in the expansion proves (3). Interestingly, this identity can be phrased as a sum over 312-avoiding permutations. Let \# $\left[i d_{n}, \sigma\right]$ denote the cardinality of the lower Bruhat interval $\left[i d_{n}, \sigma\right]$ in $S_{n}$. Then we get the following Catalan sum:

$$
\sum_{\sigma \in S_{n}(312)} \#\left[i d_{n}, \sigma\right]=(2 n-1) ! !
$$

In the next and final section, we explore how our main result can be rephrased in terms of other Catalan objects.

\section{Extension to other Catalan objects}

Throughout this exposition, we have encountered several Catalan objects: Dyck paths, non-crossing arc diagrams, and 312-avoiding permutations. The ubiquity of the Catalan numbers here and elsewhere [13, Ex. 6.19] incites further investigation into other Catalan objects to which we can relate our previous results. In particular, we express Theorem 1 both as a sum over plane forests in Section 4.1 and as a sum over binary trees in Section 4.2.

\subsection{Plane trees and plane forests}

A tree $T$ is a connected acyclic graph. A rooted tree is one possessing a unique distinguished node called the root of $T$. We consider only rooted trees and omit the adjective rooted. A node $u \in T$ is a descendant of the node $v \in T$ if $v$ is in the unique path from $u$ to the root, and $v$ is an ancestor of $u$ in this case. Thus any node $v \in T$ is both a descendant and an ancestor of itself. A descendant $u$ of $v$ is a child of $v$ if $T$ contains an edge from $u$ to $v$, in which case $v$ is the parent of $u$. A node with no children is called a leaf and an internal node otherwise. A subtree of $T$ is the tree formed by a node and all its descendants. We depict trees with child nodes situated above their parents, thus the root, being the unique ancestor to all nodes of $T$, is the bottom-most node. 
Recall that $h(v)$ is the number of descendants of $v$, and we dually define $\alpha(v)$ to be the number of ancestors of $v$. A plane tree is a tree whose subtrees are ordered linearly at each node. Denote by $\mathcal{T}_{n}$ the set of plane trees having $n$ nodes.

A plane forest is a linearly-ordered set of plane trees. Let $\mathcal{F}_{n}$ denote the set of plane forests having $n$ nodes. There is a natural bijection $\mathcal{F}_{n} \rightarrow \mathcal{T}_{n+1}$ : add a new node and make it the root of all trees in the forest $F \in \mathcal{F}_{n}$ to obtain $T \in \mathcal{T}_{n+1}$. It appears as an exercise in [13, Ex 6.19(e)] to show that the $n$th Catalan number enumerates $\mathcal{T}_{n+1}$.

The bijection $F: \mathcal{D}_{n} \rightarrow \mathcal{F}_{n}$ we consider is as follows. We construct the tree $T(\delta) \in \mathcal{T}_{n+1}$ according to $\delta$ as it is read left-to-right. We move around the nodes of $T(\delta)$ in a pre-order fashion as we construct it. Add a new edge at the current node if $\delta_{i}=e$ or move down the tree to the parent of the current node if $\delta_{i}=d$. When completed, removing the root from $T(\delta)$ completes the bijection $\mathcal{D}_{n} \rightarrow \mathcal{F}_{n}$.

Example 44. Figure 14 shows the bijection between $\mathcal{T}_{4}$ and $\mathcal{F}_{3}$, in the order corresponding to the Dyck boards shown in Figure 1.
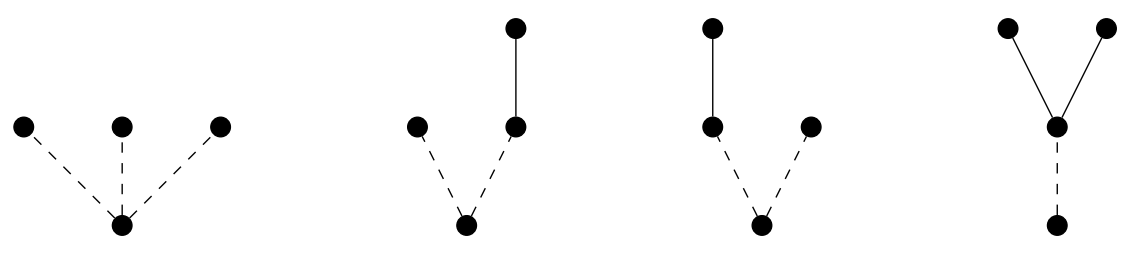

Figure 14: $\mathcal{T}_{4} \longleftrightarrow \mathcal{F}_{3}$

We now show that the rook number statistic of the Dyck board $B(\delta)$ is equal to the product of the ancestor statistic on the nodes of the corresponding plane forest $F(\delta)$ under the bijection described above.

Lemma 45. If $v_{i}$ is the ith node added to $F(\delta)$ in the above bijection $\mathcal{D}_{n} \rightarrow \mathcal{F}_{n}$, then $\alpha\left(v_{i}\right)=d_{i}(\delta)-2 i+1$.

Proof. The number $\alpha\left(v_{i}\right)$ is one more than the length of the path from $v$ to the root of the tree containing it. This is the number of steps upward less the number of steps downward until $v_{i}$ is reached. In other words, this is the number of es that appear before the $i$ th $d$ minus the number of $d$ s appearing before the $i$ th $d$ in a preorder search. Thus $\alpha(v)=\left(d_{i}(\delta)-i\right)-(i-1)$.

Proposition 46. For all $n \in \mathbb{N}$,

$$
\frac{1}{q^{n}} \sum_{F \in \mathcal{F}_{n}} \prod_{v \in F} q^{\alpha(v)}[\alpha(v)]_{q}=[1]_{q}[3]_{q} \cdots[2 n-1]_{q} .
$$

Proof. By the previous lemma the $n$th rook number $r_{n}(B(\delta))$ of the board $B(\delta)$ equals $\prod_{v \in F(\delta)} \alpha(v)$, thus we can rephrase Theorem 1 as this sum over plane forests. 
Setting $q=1$ in (5) justifies the following corollary.

\section{Corollary 47.}

$$
\sum_{F \in \mathcal{F}_{n}} \prod_{v \in F} \alpha(v)=(2 n-1) ! !
$$

In the sum over plane forests given by equation (2), the statistic $h(v)$ gives the number of descendants of the node $v$. Naturally, descendants and ancestors are structurally dual notions, but the fact that the left-hand sides of (6) and (2) are equal suggests that descendants and ancestors also have an intrinsic duality in a numerical sense.

With regard to Figure 14 in which $n=3$, equation (6) agrees with equation (3), asserting that $1+2+2+4+6=5$ !!. Equation (2) says that $6\left(\frac{1}{1}+\frac{1}{2}+\frac{1}{2}+\frac{1}{3}+\frac{1}{6}\right)=$ $6+3+3+2+1=5$ !! . Note that although the sums are equal, the sumands differ! The left-hand side of (2) is familiar in the context of binary trees, in which case the sum would be $n$ ! rather than $(2 n-1)$ !!. This observation inspires an exploration of binary trees, which is the scope of the next section. Before concluding the present section, though, we present two additional nice Catalan sums that follow from (2) by making use of the identity

$$
\frac{(2 n-1) ! !}{C_{n}}=\frac{(n+1) !}{2^{n}},
$$

which gives the proportional relationship between double-factorial numbers and the Catalan numbers. For example, applying (7) to (2), we obtain

$$
\sum_{F \in \mathcal{F}_{n}} \prod_{v \in F} \frac{2}{h(v)}=C_{n}(n+1) .
$$

With respect to the bijection $\mathcal{F}_{n} \longleftrightarrow \mathcal{T}_{n+1}$ described above, the sum (2) can be rephrased in terms of plane trees as

$$
(n+1) ! \sum_{T \in \mathcal{T}_{n+1}} \prod_{v \in T} \frac{1}{h(v)}=(2 n-1) ! !
$$

and appying (7) yields

$$
\sum_{T \in \mathcal{T}_{n+1}} \prod_{v \in T} \frac{2}{h(v)}=2 C_{n}
$$

\subsection{Binary trees}

We recursively define a plane binary tree as one whose root either has no children or whose root has a left subtree and a right subtree, each of which is also a binary tree. Let $\mathcal{P B}_{n}$ denote the set of plane binary trees with $n$ internal nodes. The removal of the $n+1$ leaves gives the bijection between plane binary trees and binary trees $\mathcal{B}_{n}$ with $n$ nodes.

The bijection $T: \mathcal{D}_{n} \rightarrow \mathcal{B}_{n}$ is as follows. Read $\delta \in \mathcal{D}_{n}$ left-to-right, and traverse $T(\delta)$ in left preorder as we construct it. The es in $\delta$ yield nodes of degree 2 , and $d$ s yield 
nodes of degree 1. At $\delta_{i}$, add two children to the current node if $\delta_{i}=e$, and add no new nodes if $\delta_{i}=d$. Continue this way until the end of $\delta$ is reached, and removing the leaves completes the bijection $\mathcal{D}_{n} \rightarrow \mathcal{B}_{n}$. To recover $\delta$, add all possible leaves to $T$ to make a binary tree, and traverse $T(\delta)$ in preorder and read each internal node as an $e$ and each leaf, disregarding the last, as a $d$.

Example 48. Figure 15 shows the five binary trees having three internal nodes, listed in the order corresponding to that of the Dyck paths in Figure 1.
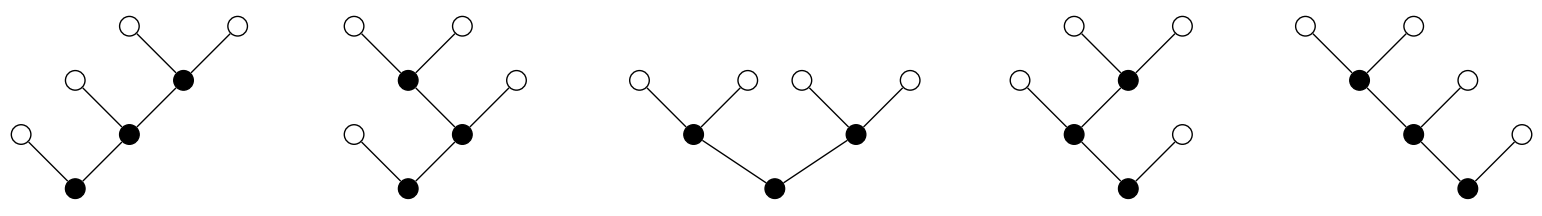

Figure 15: $\mathcal{P B}_{3}$

For the binary tree $T \in \mathcal{B}_{n}$, we define a recursive labeling $\lambda$ on its nodes. Label the root of $T$ with 1 , and label a child $u$ of $v \in T$ as follows:

$$
\lambda(u):= \begin{cases}\lambda(v)+1 & : u \text { is a left-child of } v, \\ \lambda(v) & : u \text { is a right-child of } v .\end{cases}
$$

The labeling $\lambda$ is illustrated by Figure 16 .
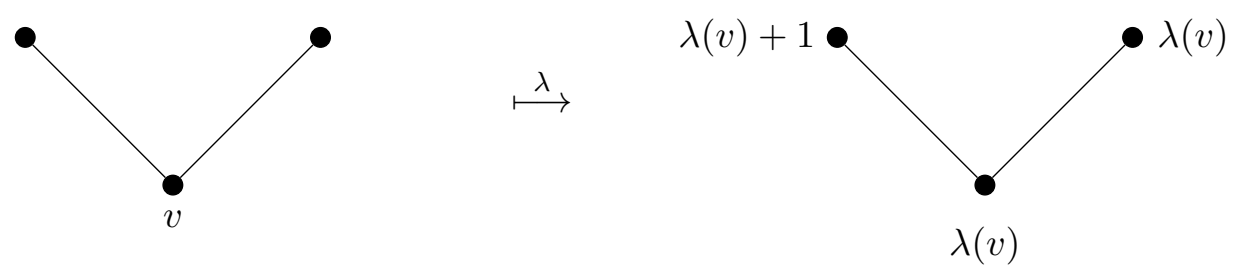

Figure 16: Labeling $\lambda$ of $T \in \mathcal{B}_{n}$.

Lemma 49. If $v_{i}$ is the ith node added to $T(\delta)$ under the above bijection $\mathcal{D}_{n} \rightarrow \mathcal{B}_{n}$, then $\lambda\left(v_{i}\right)=d_{i}(\delta)-2 i+1$.

Proof. Consider the binary subtree containing all nodes traversed in the preorder search from the root to the node $v_{i}$. At each node, we either follow an edge to a left-child or a right-child. These correspond to occurrences of $e e$ and $e d$, respectively, in $\delta$. Thus $\lambda\left(v_{i}\right)-1=d_{i}(\delta)-2 i$ counts the number of times we follow an edge to a left-child. 


\section{Proposition 50.}

$$
\frac{1}{q^{n}} \sum_{T \in \mathcal{B}_{n}} \prod_{v \in T} q^{\lambda(v)}[\lambda(v)]_{q}=[1]_{q}[3]_{q} \cdots[2 n-1]_{q} .
$$

Proof. By the previous lemma, the $n$th rook number $r_{n}(B(\delta))$ of the board $B(\delta)$ equals $\prod_{v \in T(\delta)} \lambda(v)$, thus we can rephrase Theorem 1 as a sum over binary trees.

Setting $q=1$ in (11) justifies the following corollary.

Corollary 51.

$$
\sum_{T \in \mathcal{B}_{n}} \prod_{v \in T} \lambda(v)=(2 n-1) ! !
$$

Example 52. One can verify that this holds for $n=3$ by inspection of Figure 17, which shows the five binary trees in $\mathcal{B}_{n}$ with the labeling $\lambda$ on their nodes
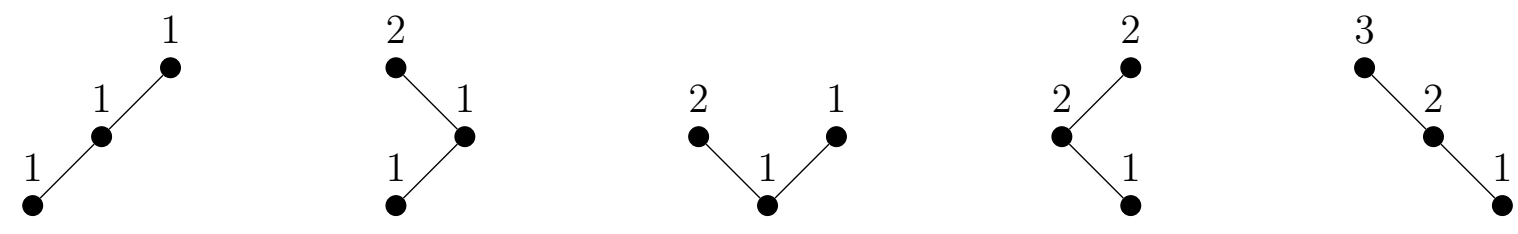

Figure 17: The labeling $\lambda$ on the nodes of the binary trees in $\mathcal{B}_{3}$

Aside from providing a combinatorial proof of (3), we have provided three natural extensions of this combinatorial identity that are expressed as sums over three other Catalan objects: 312-avoiding permutations, plane forests, and binary trees. Naturally, it would be nice to prove such expressions for other Catalan objects, such as triangulations of $(n+2)$-gons into $n$ triangles.

\section{Acknowledgments}

Most of this work was completed during the author's time at North Carolina State University. Nathan Reading is due special thanks for his his assistance, encouragement, and patience throughout the completion of this manuscript. Additionally, the author would like to thank Lou Billera and Lionel Levine for their help locating (3) in the literature.

\section{References}

[1] Louis J. Billera, Lionel Levine, and Karola Mészáros. How to decompose a permutation into a pair of labeled Dyck paths by playing a game, July 2013. arXiv: 1306.6744

[2] Anders Björner and Francesco Brenti. Combinatorics of Coxeter Groups. Springer, New York, 2005. 
[3] Anders Björner and Michelle L. Wachs. Shellable nonpure complexes and posets II (english summary). Transactions of the American Mathematical Society, 349(10):3945-3975, October 1997.

[4] Rajendra S. Deodhar and Muralik K. Srinivasan. A statistic on involutions. Journal of Algebraic Combinatorics, 13(2):187-198, 2001.

[5] Rosena R. X. Du and Fu Liu. (k,m)-Catalan numbers and hook length polynomials for plane trees. European Journal of Combinatorics, 28(4):1312-1321, May 2007.

[6] Ian P. Goulden and David M. Jackson. Combinatorial Enumeration. John Wiley and Sons, Inc., New York, 1983.

[7] Federico Incitti. The Bruhat order on the involutions of the symmetric group. Journal of Algebraic Combinatorics, 20:243-261, 2004.

[8] Donald E. Knuth. The Art of Computer Programming. Addison-Wesley Publishing Company, Reading, Massachusetts, 1973.

[9] Alexander Postnikov. Permutohedra, associaiahedra, and beyond. in: Conference in Honor of Richard Stanley's Sixtieth Birthday, June 2004. International Mathematics Research Notices, 6:1026-1106, 2009.

[10] Nathan Reading. Order dimension, strong Bruhat order and lattice properties for posets. Order, 19:73-100, 2002.

[11] Nathan Reading. Cambrian lattices. Advances in Mathematics, 205(2):313-353, October 2006.

[12] Jonas Sjöstrand. Bruhat intervals as rooks on skew Ferrers boards. Journal of Combinatorial Theory Series A, 114:1182-1198, 2007.

[13] Richard P. Stanley. Enumerative Combinatorics, Vol. 2. Cambridge University Press, Cambridge, Massachusetts, 1999. 